ÉGYPTE

monde arabe

\section{Égypte/Monde arabe}

25| 1996

Anthropologies de l'Égypte 2

\title{
L'appartenance des objets : problèmes d'anthropologie de la culture et de l'identité
}

Jean-Noël Ferrié

\section{OpenEdition}

\section{Journals}

Édition électronique

URL : https://journals.openedition.org/ema/822

DOI : 10.4000/ema.822

ISSN : 2090-7273

Éditeur

CEDEJ - Centre d'études et de documentation économiques juridiques et sociales

Édition imprimée

Date de publication : 30 mars 1996

Pagination : 15-24

ISSN : 1110-5097

\section{Référence électronique}

Jean-Noël Ferrié, «L'appartenance des objets : problèmes d'anthropologie de la culture et de

l'identité », Égypte/Monde arabe [En ligne], 25 | 1996, mis en ligne le 08 juillet 2008, consulté le 07 juillet 2022. URL : http://journals.openedition.org/ema/822 ; DOI : https://doi.org/10.4000/ema.822

Ce document a été généré automatiquement le 7 juillet 2022.

Tous droits réservés 


\title{
L'appartenance des objets : problèmes d'anthropologie de la culture et de l'identité
}

\author{
Jean-Noël Ferrié
}

1 Je partirai de l'affirmation d'un anthropologue, Peter Geschiere. Celui-ci écrit : « II est indéniable que des groupes périphériques s'engagent de plus en plus dans le marché mondial, non seulement comme producteurs, mais surtout comme consommateurs, de sorte que les nouvelles vogues et les derniers gadgets apparaissent partout. Le paradoxe est pourtant que ces processus ne semblent guère mener à une uniformité culturelle croissante, ou à la victoire d'une culture mondiale du Coca-Cola, redoutée par Lévi-Strauss. Au contraire, l'hétérogénéité culturelle de la société mondiale moderne semble s'approfondir. $»^{1}$ Cette façon de voir conduit souvent les anthropologues à expliquer que l'usage marocain du téléphone est strictement marocain, que la conception égyptienne de la Mercedes est seulement égyptienne et que boire du CocaCola au Caire n'a rien à voir avec le fait d'en boire à Minneapolis. En d'autres termes, ils défendent l'idée selon laquelle les objets et les références venues de l'«ailleurs » subissent un encodage local qui en fait des objets et des références du lieu. En revanche, il semble qu'ils négligent (sans véritablement le méconnaître) un phénomène non moins important : le fait que cet encodage installe aussi le local dans l'«ailleurs », c'est-à-dire l'accommode à la différence.

Il est possible d'expliquer les raisons de ce choix ; éviter à la fois l'erreur des théories de l'homogénéisation et l'erreur du culturalisme ${ }^{2}$. Il est, certes, aisé de comprendre en quoi les théories de l'homogénéisation sont contredites par ce point de vue, mais il est certainement plus difficile de comprendre en quoi le culturalisme est évité. En fait, cela provient de ce que le terme d'encodage sert à désigner le moteur d'une acculturation permanente, c'est-à-dire de la production d'une différence culturelle «toujours différente", là où le culturalisme ne voyait que des cultures réfractaires au changement et immobiles. Néanmoins, le reproche que je ferais à cette conception est d'oublier que ce jeu de différenciations ne peut être pris en considération 
indépendamment du processus de synchronisation culturelle qui l'accompagne, surtout quand il s'agit d'expliquer que les cultures locales et leurs agencements sociaux se situent dans la modernité.

3 La modernité, en effet, ne consiste pas dans l'encodage local des références et des valeurs de l'« ailleurs » (elle ne réside pas, par exemple, dans l'arabisation du software\}. Cette performance produit, certes, quelque chose que l'on pourrait nommer le " changement social», mais ne produit pas la modernité. Car celle-ci est, avant tout, une limitation des droits du local comme de ses compétences. En d'autres termes, je ne crois pas que la définition de la modernité soit l'encodage du neuf, c'est-à-dire sa prise de possession par le local ou la capacité du local à intégrer la novation à son système de référence.

Ce point de vue m'éloigne de la position défendue, par exemple, par Fariba Adelkhah à propos de l'encodage des machines à laver en Iran. L'argument qu'elle développe est le suivant; en Iran, on sépare les linges considérés comme souillés de ceux qui ne le sont pas, c'est-à-dire que l'on procède, pour le lavage en machine, comme pour le lavage «traditionnel » à la main, en suivant une logique culturelle de distinction. Pour elle, ceci est la preuve que la machine à laver est, dans son entièreté, inscrite dans un monde spécifique qu'elle ne détermine pas. Ce qui est intrinsèquement gênant dans cette conception tient à l'idée que la sphère culturelle - ce que l'on nomme aussi l'identité devient la matrice forcée de tout changement, c'est-à-dire que l'accès au reste du monde se fait forcément par la médiation du local. Je crois que cette idée est erronée et que, par ce biais, la bonne volonté culturelle des anthropologues - et, plus largement, des social scientists - aboutit paradoxalement à justifier des positions «identitaristes " fortes prônant la soumission des individus à un ordre culturel intangible. La question de l'appartenance des objets et des processus cognitifs décidant de cette appartenance s'avère ainsi - sous son apparence quelque peu incongrue - au centre de nombreux débats sociétaux. Je voudrais, dans les pages qui suivent, montrer - à l'aide de quelques exemples égyptiens- combien est erronée l'idée d'encodage, au sens d'une domination inévitable et nécessaire - de l'interprétation locale des ressources d'un objet.

Les limites de l'appartenance des objets

5 Les logiques de réappropriation et donc d'appartenance sont d'abord limitées par la nature même des objets. Si l'on peut à juste titre critiquer l'idée selon laquelle la généralisation du Coca-Cola ressortit de l'homogénéisation du monde, il paraît tout autant excessif de prétendre que la bouteille de Coca-Cola consommée au Caire l'est dans l'ignorance des centaines d'images installant cette bouteille dans la mythologie moderne. Je ne boirai certainement jamais du Coca-Cola comme un cow-boy du Montana, pourtant cette différence évidente n'empêchera pas le Coca-Cola d'être au Montana, en Égypte et dans d'autres lieux, un même produit qu'un Américain en voyage au Caire reconnaîtra et qu'un Égyptien de passage pourra consommer sans étonnement dans un motel des Rocheuses. Je crois qu'une part des problèmes (douloureux) que nous nous posons vis-à-vis de la dynamique locale des objets transnationaux disparaîtrait si nous ne confondions pas la biographie des utilisateurs (leur subjectivité vis-à-vis de l'objet) et la biographie de l'objet lui-même (ce qu'il est objectivement, quoique j'en pense ou qu'en pense l'homme du Montana). Un objet possède en effet en propre sa matérialité, et cette matérialité impose des contraintes (ainsi que des conduites) et propose des conduites (ainsi que des contraintes) 
indépendamment de la subjectivité des usagers et de leur façon "culturelle » de construire le monde.

6 Prenons comme exemple la façon dont les Égyptiens considèrent les Mercedes. En tant qu'anthropologue, je peux aisément procéder à une description en termes de valeurs culturelles et aboutir, après réduction des rationalités techniques, au genre de conclusion à laquelle parvient Geertz avec le combat de coqs balinais ${ }^{3}$ : donner une idée du monde subjectif dans lequel vivent les Égyptiens, interpréter une forme de vie. À vrai dire, je ne crois pas que la structure subjective soit la vérité ultime du combat de coqs et je ne suis pas sûr que l'on puisse adopter aussi facilement (sans être quelque peu culturaliste) une perspective hiérarchique entre les niveaux. Certes, je puis dire que le culte de la Mercedes (et, de plus en plus, celui de la BMW) révèle l'importance des conduites ostentatoires dans la société égyptienne et témoigne de son ethos. D'une certaine façon, posséder une Mercedes, c'est être un certain type d'homme et imposer une certaine forme de respect. Mais je puis faire la même remarque en me transportant en France ou en Italie dans la mesure où, en tant que réalisation technique destinée à maintenir la réputation d'excellence de la marque, ces voitures ont un coût important. Cette qualité implique aussi que l'on puisse faire avec une Mercedes des choses que l'on ne ferait pas avec une autre voiture (ou avec une voiture de moindre importance). $\mathrm{Si}$, sur le pont du Six-Octobre au Caire, une Mercedes double mon taxi et toute une série d'automobiles en klaxonnant impatiemment, ce n'est pas seulement parce que le conducteur appartient à une frange particulièrement sûre d'elle-même de la bourgeoisie, mais aussi parce que sa voiture lui permet ce genre d'accélération. En d'autres termes, les potentialités intrinsèques de l'objet déterminent en partie le type de réappropriation auxquelles il peut donner lieu, et le type de réappropriation luimême dépend, en partie, de sa propre biographie, c'est-à-dire des significations qu'il a acquises en d'autres lieux mais qui sont constitutives de son image, où qu'il soit : la Mercedes est une voiture de luxe dans sa conception, aussi peut-elle se prêter à des stratégies d'ostentation. Remarquons, en outre, que la conception « locale » du luxe se conforme ici à un standard transnational. Certes, je peux expliquer que le prestige social passe par la possession d'un certain style de voiture, que la voiture est l'équivalent fonctionnel d'un cheval de prix ou d'une concubine circassienne mais il me serait, je crois, assez difficile d'affirmer que l'ensemble de la voiture figure dans cette évaluation culturelle. D'une certaine manière, quelque chose d'elle y figure effectivement, mais ce n'en est pas la totalité. Un découpage de l'objet voiture est conforme à cette interprétation (tout au moins peut-on l'admettre), mais d'autres découpages sont possibles, qui ont assez peu à voir avec celle-ci. En ce sens, la localisation, l'encodage ou la réappropriation ne concernent que des sections virtuelles de l'objet.

Les objets multiréférencés

$7 \quad$ La possibilité de ce découpage provient de ce que les objets appartiennent toujours au système de référence dans lequel ils sont créés. L'encodage n'est en aucune manière capable d'obvier à cette appartenance première. Prenons par exemple les vêtements, et quelque chose qui a fort à voir avec ce que l'on voudrait définir comme une tradition culturelle : la pudeur féminine. Je ne reviens pas sur le fait que de telles dénominations - «tradition culturelle » - n'ont aucune réalité anthropologique ; elles sont seulement et ne seront jamais autre chose que des étiquetages d'acteurs. En ce sens, les objets, les conduites et les valeurs qu'elles désignent ne relèvent pas d'un espace spécifique - le «monde de la tradition » - antagonique à la modernité. La structure d'expression et de 
validation desdites traditions est d'ailleurs exactement la même que celles des novations vilipendées par les "petits entrepreneurs de morale »4. Prenons l'un des magazines égyptiens spécialisés dans la mode pour femmes voilées. Il s'intitule «Élégance et pudeur" (Anâqa wa hishma) et présente exclusivement des modèles accompagnés d'un accessoire - foulard, chapeau et foulard, toque et foulard... dissimulant la chevelure. Les mannequins photographiés ne sont pas exactement semblables à ceux que l'on peut voir dans des revues internationales comme Vogue ou dans des revues égyptiennes relevant du même «look» comme Pose. Ils présentent un certain embonpoint, ne montrent que leurs visages, leurs mains et, parfois, leurs chevilles. Le maquillage est à la fois présent et sobre (par rapport aux habitudes égyptiennes), jouant sur les tons clairs et pâles (à l'exception des sourcils et des cils). La plupart des vêtements sont «occidentaux»: tailleurs, ensembles, vestes croisées, spencers, robes, pantalons, jupes, chemisiers, pull-overs. Ils sont présentés dans un décor cossu, lui-même « occidental » : meubles de style Empire ou Louis XVI, cheminées en briques aux formes géométriques. Le style d'ensemble est incontestablement égyptien, malgré l'origine totalement étrangère du modèle des vêtements et des meubles.

8 Mais qu'appelle-t-on alors le style "égyptien" en matière de mode? On ne peut ignorer qu'il est, ici, uniquement constitué d'éléments étrangers. Certes, il est possible de repérer ce qui; dans ces éléments, est transformé conformément au goût « local » et y voir la preuve d'une " appropriation » ou d'un « encodage ", ainsi que le soutiennent maints anthropologues travaillant sur la «biographie» des produits transnationaux. Toutefois, ce point de vue me semble se heurter à une objection majeure : le fait que l'opération nommée encodage ne puisse être décrite comme une pure et simple localisation de produits (ou de valeurs, de références...) étrangers. Même en conservant l'idée de "localisation ", il convient de noter que le processus est double : il installe le «transnational» dans le «local» mais, en même temps, situe le «local» dans le «transnational». Car nous n'avons jamais affaire à une coûteuse opération de traduction aboutissant à la recréation locale des produits transnationaux; nous avons plutôt affaire à ce que l'on pourrait nommer, suivant Bourdieu ${ }^{5}$, une opération d'homologation. Cette opération ne consiste pas à transformer radicalement les produits étrangers mais, plus simplement, à ajouter ou à supprimer certaines de leurs caractéristiques afin de les rendre compatibles avec les divers goûts - c'est-à-dire avec les esthétiques et les éthiques - en cours dans la société. Ni les uns ni les autres ne sont d'ailleurs spécifiquement différents de ceux présents dans d'autres sociétés. Ce que l'on pourrait nommer la matrice des goûts en usage dans un ensemble social comprend bien des choses également considérées comme belles et souhaitables ailleurs. Ainsi peut-on aimer porter le voile avec un spencer et une jupe. Le goût pour le spencer et la jupe ne sont en rien "égyptiens ", Ils proviennent de la circulation internationale de modèles. On peut effectivement avancer que le foulard « localise » l'ensemble de la toilette, mais il sera difficile d'affirmer que le système de sens dont il relève a quelque chose à voir même de façon lointaine - avec celui qui détermine la pertinence du port du spencer pour une femme, avec une pochette comme accessoire. En d'autres termes, le système de sens introduit par le voile est incommensurable avec le système de sens de la toilette.

9 Par incommensurable, je ne veux pas souligner une contradiction qui s'exprimerait dans l'ordre même du paraitre en public ou dans l'élégance du modèle; j'entends seulement indiquer que le voile n'indexe pas la toilette, qu'il l'homologue seulement et 
que, ce faisant, il ne crée pas un autre univers. Ainsi, les modèles présentés dans "Élégance et pudeur » ont comme univers de référence le système international de la mode. Outre le fait qu'ils sont mis en scène dans des photographies de mode respectant les principaux critères du genre, leur environnement publicitaire est celui des " grands couturiers » : Christian Dior, Balmain... Les femmes figurant dans ces publicités ne sont pas voilées et ne sont pas non plus exactement pudiques (même si elles sont élégantes), puisque ce sont les mêmes que celles que l'on retrouve dans les revues comme Vogue. La succession indifférenciée de femmes "pudiques» et de femmes qui ne le sont pas suggère que la norme de représentation en cours dans une publication aussi volontairement austère que "Élégance et pudeur " n'est pas quelque chose qui serait précisément la " pudeur égyptienne » mais, bel et bien, la mode internationale. C'est à l'intérieur de ce système que les stylistes de la revue tentent de définir un espace local spécifique. Il est cependant clair que ce local n'est pas, ici, l'expression d'une spécificité préexistante mais son invention dans un contexte qu'il ne détermine pas et dont on peut même considérer qu'il est largement délocalisé. L'élégance pudique égyptienne se crée ainsi par rapport au restant de la mode (et du monde), c'est-à-dire au-delà de la référence à un territoire ou à une communauté territorialisée ${ }^{6}$.

La question de l'homologation

10 II doit maintenant être bien clair que le « local» est le produit d'une homologation et non la conséquence de la conformité à une tradition culturelle. Il doit aussi être clair que l'homologation a quelque chose à voir avec la dissimulation de l'ouverture de la référence - c'est-à-dire de la novation - et sa disjonction d'avec le territoire. Prenons comme exemple le port du voile en Égypte et la façon dont Târiq al-Bishri le justifie : "Où se situe le point de divergence entre ses adeptes et ses détracteurs? La mentalité occidentale préfère poser le différend en termes de progrès et d'arriération. Le higâb serait ainsi l'expression du mauvais traitement réservé à la femme. (...) Où se situe l'essence de la question du voile? Plutôt que dans le voile en tant que tel, ne serait-ce pas dans l'éducation et le travail de la femme? Qui oserait prétendre que le fait de se couvrir ta tête et de rallonger ses vêtements soit un obstacle aux études et à l'exercice d'une profession? (...) Mais non : au lieu d'être posé en termes de salubre controverse, de discussion - l'enjeu étant l'accès de la femme à l'éducation et à l'enseignement -, la question est posée pour les musulmans en des termes qui portent atteinte à leurs croyances essentielles et par contre, pour l'Occident laïc, elle est posée en termes de modèle de comportement et de référence de vie, de jugement de valeur. L'insistance à poser le problème en termes vestimentaires et non en termes d'éducation et de travail correspond en fait à une volonté d'aborder la question sous l'angle des cadres de référence... $»^{7}$

11 Que signifie ceci? Que l'acceptation de la novation (l'instruction féminine, le travail des femmes) diffère nettement de la reconnaissance de la possibilité de choisir les cadres de références pour évaluer les conduites et les attitudes. Novation et ouverture de la référence peuvent ainsi être parfaitement posées comme deux choses séparées. Certes, on demeure libre de penser que la novation importe - en fraude, pourrait-on dire d'autres systèmes de référence, mais ce point de vue ne change rien à la possibilité d'en faire abstraction et de maintenir la fiction de l'unicité de la référence. Cette fiction a des effets sociaux dans la mesure où elle impose une limite au droit effectif (et non pas seulement subjectif) de faire du neuf, puisque toute novation se doit alors de respecter

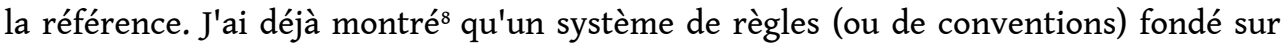
une référence unique (et transcendante) - la Loi divine - limitait, de fait, les possibilités 
de novation des acteurs en les dépossédant de la ressource d'ouvrir leurs systèmes de choix sans tenir compte du respect des formes, au sens où l'entend Bourdieu : « Mettre les formes, c'est donner à une action ou à un discours la forme qui est reconnue comme convenable, légitime, approuvée, c'est-à-dire une forme telle que l'on peut produire publiquement, à la face de tous, une volonté ou une pratique qui, présentée autrement, serait inacceptable... »"

Certes, il est assez souvent possible d'y mettre les formes mais, dans bien des situations, cela devient rapidement impossible, soit parce que l'action contredit explicitement les principes de forme eux-mêmes (se promener en topless une plage d'Alexandrie, par exemple), soit parce que le respect de la forme conduit à respecter (tout au moins, plus longtemps que dans d'autres situations) le contenu dont la forme est censée n'être que l'apparence (aller jusqu'à s'abstenir de relations sexuelles prohibées pour ne pas être soupçonné d'en avoir). Dans tous les cas relevant de ces catégories, la nonreconnaissance du droit de faire du neuf - c'est-à-dire d'avoir des conduites justifiées dans d'autres systèmes de références - a pour conséquence d'interdire toute une série de comportements. Pour donner un rapide exemple, la possibilité de porter le Jean sous le higâb n'empêche pas de porter le jean mais empêche de se promener en jean, et je ne crois pas que l'on puisse affirmer que cette interdiction soit sans effets sur le rapport à soi et sur la relation à autrui (tout particulièrement en ce qui concerne les relations intersexuelles).

En ce sens, une novation n'est pas forcément connectée à une conduite novatrice, c'està-dire à une conduite exprimant le droit de faire du neuf. On peut même considérer qu'une partie du travail de codification des conduites nouvelles est moins un travail d'encodage - au sens plutôt neutre d'adaptation des objets ou des valeurs de l'« ailleurs » aux spécificités locales - qu'un travail d'homologation, c'est-à-dire une euphémisation de leur contenu novateur. Cette euphémisation consiste à leur ôter tout caractère apodictique propre (la nouveauté ne peut tirer des conditions de sa production la source de sa légitimation), à les contraindre à n'exister que sous une certaine forme. Il s'agit moins d'une opération de traduction que d'une reformulation (d'un recadrage) limitée. Prenons les vidéo-clips de Samira Saïd (une chanteuse marocaine faisant carrière en Égypte). Ceux-ci sont principalement mis en scène en conformité avec l'esthétique des vidéo-clips tournés en Europe ou aux États-Unis. Ainsi, dans l'un d'eux, on voit la chanteuse entrer dans un bar américain dont les clients sont seulement des hommes... mais où le barman sert du jus d'orange. L'opération d'homologation ne consiste, ici, qu'à remplacer l'alcool par du jus d'orange, à respecter un critère de forme qui altère d'autant moins le sens général de la scène qu'il est toujours possible de la rétablir dans sa version "normale », c'est-à-dire d'oublier le jus d'orange.

14 Quand on parle d'homologation au lieu d'encodage, il devient clair que l'objet de la localisation n'est pas de reconstruire le monde de l'« ailleurs » dans les termes locaux, de le traduire en gommant en même temps la version originale. Deux opérations peuvent ainsi être distinguées ;

- la « localisation » limite les registres expressifs des conduites, des conceptions éthiques, des objets ou des modes sans pour autant rendre impossible la recomposition de leurs registres originaux, le monde dans lequel ils ont été conçus (la scène du bar avec le jus d'orange n'est ainsi lisible que parce que l'on remplace presque inconsciemment le jus d'orange par de l'alcool $)^{10}$; 
- elle les met, en même temps, en conformité avec ce que le « local » reconnaît comme le fondement de sa spécificité (ses valeurs centrales de cohésion, en quelque sorte). En ce sens, je crois que l'on peut considérer que l'homologation met en œuvre un « encodage » restreint dont l'enjeu est la dissimulation de l'ouverture de la référence et le fait que les objets appartiennent précisément à un système de référence et à des réseaux de diffusion plutôt qu'à un territoire, c'est-à-dire à une identité localisée. L'homologation sert à donner l'image d'un ordre qui déjà n'existe plus. Il nous faut certainement penser l'appartenance comme autre chose qu'une localisation. Et ce qui vaut pour les objets vaut, bien sûr, pour les humains...

\section{NOTES}

1. Geschiere P., Sorcellerie et politique en Afrique, Karthala, Paris, 1995, p. 15.

2. Par culturalisme, $\mathrm{j}$ 'entends la doctrine anthropologique qui considère qu'une action $\mathrm{A}$ de $\mathrm{X}$ est explicable par les valeurs de la société de $\mathrm{X}$ qui sont à même de rendre compte, à elles seules, des motivations et de la .fonctionnalité de l'action A. La culture établirait ainsi un monde clos de motivations.

3. Geertz C., « .Le combat de coqs balinais », Bali. L'interprétation d'une culture, Gallimard, Paris, 1983.

4. Suivant Becker H., Outsiders, Métaillé, Paris, 1985.

5. Bourdieu P., « La codification », Choses dites. Minuit, Paris, 1987.

6. Ceci ne vaut pas que pour la mode pudique égyptienne. Ce que l'on nomme la « mode française » et dont on fait volontiers un signe de l'excellence de la France est encore plus largement une création du restant du monde. La nette présence de l'anglais comme koiné dans les coulisses des défilés de mode, comme la présence marquante de créateurs étrangers dans les maisons de couture françaises, suggère bien que la mode française est actuellement plutôt le fruit d'un consensus et d'un travail international que du seul « génie » français.

7. Cf. Burgat F., « Les conditions d'un dialogue avec l'Occident : entretien avec Târiq al-Bishri », Égypte/Monde arabe $\mathrm{n}^{0} 7,3^{\mathrm{e}}$ trimestre 1991.

8. Cf. Ferrié J.-N., « Réformisme, renaissance et contingence : la justification de la justice chez Nubar pacha et Khayr al-Din », La Réforme sociale en Égypte (dir. A. Roussillon), Cedej, Le Caire, 1995.

9. Bourdieu P., op. cit., p. 103.

10. Dans la mesure où les instances d'homologation ne peuvent contrôler effectivement la circulation de l'information, mais seulement éviter certaines de ses conséquences sur la structuration de l'espace public. 
INDEX

Mots-clés : culture, identité, anthropologie

\section{AUTEUR}

JEAN-NOËL FERRIÉ

Cedej 